\title{
RECHERCHES DE CHIMIOTHÉRAPIE SUR LA STRONGYLOÏDOSE DES RATS (1)
}

\author{
Par Albert ERHARDT et KarI DENECKE
}

La strongyloïdose des rats a déjà été utilisée par Arreaza-Guzman (1935) pour l'épreuve de préparations anthelmintiques. L'agent de cette infection est le Strongyloides ratti Sandground, dont la biologie et la position systématique ont été bien décrites par ArreazaGuzman (1935) en tenant compte de tous les travaux parus à ce sujet. Depuis la publication de ce travail, l'évolution et le pouvoir infectieux du Strongyloides ratti ont notamment fait l'objet de nouvelles recherches de la part de Graham (1936-1938) et de Sheldon (1936-1938).

Arreaza-Guzman a eu des résultats négatifs avec les pyréthrines, le violet de gentiane, le thymol, l'essence de chénopode, le tétrachloréthylène et la glycérine. Nous avons essayé à notre tour le pouvoir vermicide de la "bédermine », combinaison d'ascaridol et seretin (tétrachlorure de carbone), sur la-strongyloïdose des rats. Le médicament a été aimablement mis à notre disposition par la « I.G. Farbenindustrie, Werk Elberfeld 》.

Les rats ont été soumis à une infection par la peau avec des larves filariformes de Strongyloides ratti. Nous avons utilisé, pour nos expériences, la souche de Strongyloides avec laquelle ArreazaGuzman avait déjà travaillé et qui avait été isolée d'un rat, [souche Brumpt 689-XIV, isolée à partir d'un lot de surmulots de Ménilmontant (Paris)], par le prof. Brumpt. Nous avons employé, pour nos cultures et nos infections, les méthodes de Brumpt, en usage à l'Institut de Parasitologie de la Faculté de médecine de Paris et déjà décrites par Arreaza-Guzman. Par contre, nous avons combiné une nouvelle méthode quantitative de numération des œufs dont voici la description :

Les déjections de 24 heures des rats sont d'abord broyées soigneusement dans un petit mortier avec un peu de solution à 1 p. 100 de carbonate de sodium. On ajoute ensuite une quantité suffisante

(1) Traduit de l'allemand par le $\mathrm{D}^{r}$ Maurice Langeron.

Anfales de Parasitologie, T. XVII, $\mathrm{N}^{\circ} 3 .-1^{\text {er }}$ mai 1939 , p. 199-205. 
de cette solution pour obtenir une bouillie fécale assez claire. Cette bouillie, qui renferme tous les œufs de Strongyloides émis en 24 heures, est versée dans l'appareil de Zschucke que l'on remplit

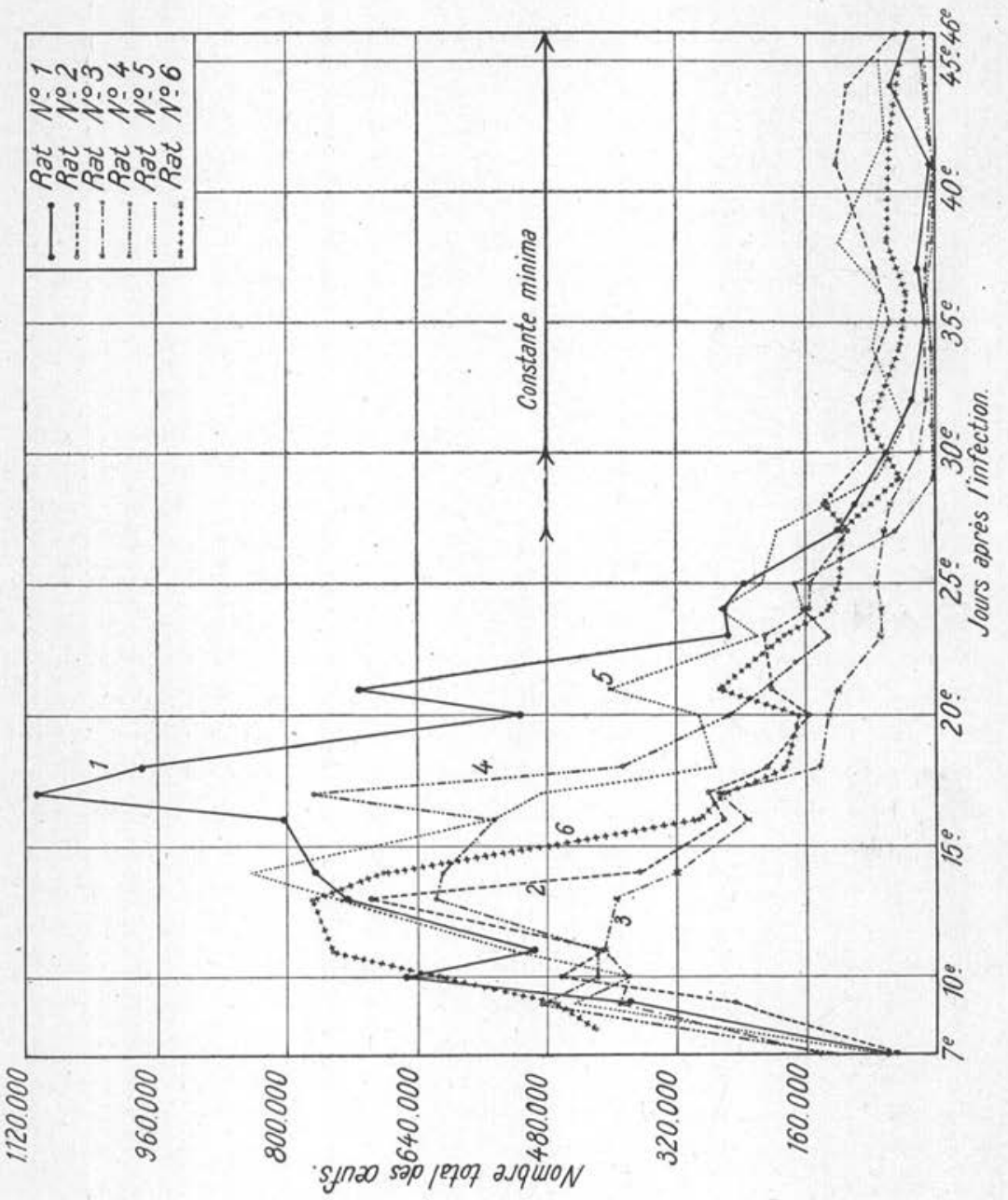

ensuite jusqu'au trait supérieur $\left(60 \mathrm{~cm}^{3}\right)$ avec la solution de carbonate de sodium à 1 p. 100 . On agite ensuite fortement cet appareil qui renferme 10 billes de verre; ensuite, on puise avec une pipette un peu de la bouillie fécale qui ne doit plus tenir en suspension de particules grossières et on en introduit une quantité 
déterminée dans la chambre à compter de Zschucke (1931). Pour ces recherches, il suffit de faire la numération des œufs dans la moitié de la chambre à compter $\left(0 \mathrm{~cm}^{3} 0375\right)$. Le résultat moyen de plusieurs numérations doit être multiplié par 1.600 pour qu'on obtienne le nombre total des œufs présents dans les déjections.

Nous avons d'abord établi le cours normal de la ponte, du $7^{\circ}$ au $46^{\circ}$ jour après le jour de l'infection, chez six rats (voir le graphique). Déjà, au $7^{\circ}$ jour, nous avons trouvé chez tous les rats de nombreux œufs dans les crottes ; pourtant, d'après les recherches de Graham (1936), le commencement de la ponte oscille entre le $5^{\circ}$ et le $11^{\circ}$ jour. Nos courbes montrent d'abord une forte montée du nombre des œufs. Le maximum se trouve, chez les divers rats, entre le $13^{\circ}$ et le $17^{\circ}$ jour (inclus) après le jour de l'infection. Après ce maximum, le nombre des œufs s'abaisse plus ou moins rapidement jusqu'aux environs du $30^{\circ}$ jour. Dans les jours suivants, le nombre des œufs reste à peu près au même niveau. Cette « constante minima 》 dure au moins jusqu'au $46^{\circ}$ jour, moment auquel nous avons arrêté nos numérations; le début de la « constante minima » a lieu au plus tôt au $27^{\circ}$ et au plus tard au $30^{\circ}$ jour après le jour de l'infection. On ne peut évidemment pas attribuer l'allure de la courbe à ce que chaque ver produit d'abord un grand nombre d'œufs qui diminue ensuite de plus en plus; il est, au contraire, très vraisemblable que les vers meurent suivant l'abaissement de la courbe et qu'un faible pourcentage $(1,2-1,5$ p. 100) reste plus longtemps vivant (au-delà de 30 jours) (Sheldon, 1937). Les Strongyloides morts ne se retrouvent pas plus dans les déjections que les Opisthorchis tués (Erhardt, 1932).

D'après le nombre d'œufs pondus pendant la période de la « constante minima » et d'après celui des vers trouvés à l'autopsie, on peut calculer combien d'œufs environ une femelle parthénogénétique pond par jour et combien de vers correspondent à chaque wuf compté. Le tableau I montre les résultats de nos recherches à ce sujet.

Il y a donc en moyenne de 2 à 10 vers pour chaque œuf compté dans la moitié de la chambre ; on peut en déduire immédiatement le chiffre moyen de l'intensité de l'infection. Chez les divers rats, le nombre des œufs pondus chaque jour par une femelle de Strongyloides ratti pendant la période de «constante minima » varie donc entre 175 et 1.000 . Comparativement, nous rappelons que le nombre d'œufs pondus chaque jour par une femelle d'Ancylostoma canimum (Ercolani) du chat atteint de 953 à 9.500 (Erhardt, 1938), tandis que, pour Opisthochis tenuicollis (Rud.) (Erhardt, 1935; 
TABLEAU I

Ponte journalière des femelles de Strongyloides ralli et rapports entre le nombre des œufs et celui des vers pendant la période constante minima

\begin{tabular}{|c|c|c|c|c|c|c|c|c|}
\hline RAT $\mathrm{N}^{*}$ & 1 & 2 & 3 & 5 & 6 & 12 & 13 & 14 \\
\hline Poids en gr..... & 165 & 85 & 220 & 60 & 65 & 175 & 150 & 255 \\
\hline $\begin{array}{l}\text { Début de la cons- } \\
\text { tante minima } \\
\text { (nombre de } \\
\text { jours aprèscelui } \\
\text { de l'injection).. }\end{array}$ & 30 & 27 & 30 & 29 & 29 & $\begin{array}{c}(30) \\
\text { (non som }\end{array}$ & $\begin{array}{c}\text { (30) } \\
\text { aptés aup }\end{array}$ & $\begin{array}{c}(30) \\
\text { aravant) }\end{array}$ \\
\hline $\begin{array}{c}\text { Nombre des jours } \\
\text { de détermina- } \\
\text { tion........... }\end{array}$ & 7 & 9 & 7 & 7 & 7 & 9 & 3 & 10 \\
\hline $\begin{array}{r}\text { Moyenne des œufs } \\
\text { comptés par } \\
\text { jour........... }\end{array}$ & 19,4 & 52,0 & 7,6 & 44,7 & 32,4 & 67,8 & 11,2 & 28,6 \\
\hline $\begin{array}{c}\text { Calcul du total des } \\
\text { œeufs par jour. }\end{array}$ & 31040 & 83200 & 12160 & 71520 & 51840 & 108480 & 17920 & 45760 \\
\hline $\begin{array}{c}\text { Nombre de vers } \\
\text { trouvés à l'au- } \\
\text { topsie.......... }\end{array}$ & 116 & 106 & 24 & 200 & 203 & 625 & 18 & 134 \\
\hline $\begin{array}{r}\text { Nombre de vers } \\
\text { pour un œuf } \\
\text { compté......... }\end{array}$ & 6 & 2 & 3 & 4 & 6 & 9 & 2 & 5 \\
\hline $\begin{array}{r}\text { Nombre d'œufs } \\
\text { pondus par jour } \\
\text { parune femelle. }\end{array}$ & 268 & 785 & 507 & 358 & 255 & 174 & 995 & 341 \\
\hline
\end{tabular}

Ejsmont, 1937), la constante moyenne journalière est à peu près de 880 œufs (Erhardt, 1933).

De ces recherches, nous avons déduit une méthode de contrôle pour les recherches de chimiothérapie sur la strongyloüdose des 
rats: les rats doivent être infestés aussi massivement que possible avec plusieurs milliers de larves filariformes de Strongyloides ratti (pour les méthodes quantitatives d'infection, voir Erhardt, 1938). La numération des œufs doit commencer au $30^{\circ}$ jour de l'infection. $\mathrm{Au} 33^{\circ}$ jour, le médicament est administré et la ponte est à nouveau suivie. Au bout d'environ 8 à 10 jours, les animaux en expérience et les animaux témoins sont tués et autopsiés. De la forme de la courbe, du nombre quotidien d'œufs et du résultat de l'au-

TABLEAU II

Recherches de chimiothérapie sur la strongyloïdose des rats. Chaque rat a reçu, una fois, par la bouche, $5 \mathrm{~cm}^{3}$ de solution de bédermine par kilog. II n'y a pas eu d'effet vermicide.

\begin{tabular}{|c|c|c|c|c|c|}
\hline $\operatorname{RAT} \mathbf{N}^{*}$ & 8 & 9 & 10 & 11 & 12 \\
\hline Poids en gr............. & 160 & 200 & 210 & 130 & 175 \\
\hline $\begin{array}{c}\text { Moyenne des œufs comptés } \\
\text { par jour .................... }\end{array}$ & 57,0 & 32,7 & 25,7 & 42,5 & 67,8 \\
\hline Calcul des vers........... & $114-570$ & $65-327$ & $51-257$ & $85-425$ & $123-678$ \\
\hline Vers trouvés vivants...... & 274 & 141 & 67 & 162 & 625 \\
\hline $\begin{array}{l}\text { Nombre de jours entre } \\
\text { l'administration de la bé- } \\
\text { dermine et l'autopsie... }\end{array}$ & 2 & 9 & 9 & 9 & 8 \\
\hline Effet visible.............. & neant & neant & neant & & neant \\
\hline
\end{tabular}

topsie, on déduit l'activité du médicament. A l'autopsie, il faut examiner au microscope le raclage du contenu intestinal. Les vers se trouvent surtout dans le duodénum, donc dans le premier quart des 80 centimètres d'intestin qui se trouvent entre l'estomac et le cæcum.

Nous avons essayé l'action de la «bédermine » sur la strongyloïdose des rats. Nous avons employé, pour ces recherches, la solution de "bédermine » à 14 p. 100 dans l'huile de ricin, qui se trouve dans le commerce ; la «bédermine» est une combinaison d'ascaridol et de seretin dans le rapport $1: 6$. Cette combinaison a déjà été essayée dans une proportion analogue par Erhardt (1938) pour l'ancylostomose du chat. On ne peut prouver une augmentation de puissance de la combinaison tétrachlorure de 
carbone-ascaridol, mais au moins, dans un cas, l'action s'est montrée plus efficace.

Les rats recevaient, par voie buccale, $5 \mathrm{~cm}^{3}$ de solution de « bédermine » par kilogramme, dose qui, pour une partie des animaux, était déjà mortelle. Le tableau 2 montre les résultats que nous avons obtenus.

\section{RÉSUMÉ}

1. Description d'une méthode quantitative de contrôle pour anthelmintiques dans la strongyloïdose des rats.

2. Pendant la période de « constante minima », qui commence au plus tard le $30^{\circ}$ jour de l'infection et dure au moins jusqu'au $46^{\circ}$ jour, la ponte journalière des œufs de Strongyloides ratti est à peu près uniforme. Le nombre des œufs pondus chaque jour par une femelle de Strongyloides pendant cette période varie, suivant les rats, entre 175 et 1.000 . C'est pendant cette période que nos essais de chimiothérapie ont été effectués.

3. La "bédermine », combinaison d'ascaridol et de seretin (tétrachlorure de carbone), est, même à dose élevée, sans effet vermicide sur le Strongyloides ratti: nous n'avons pas pu une seule fois constater une action sur la ponte.

\section{BibLIOGRAPHIE}

Arreaza-Guzman (A.). - Contributior expérimentale d̀ l'élude du traitement de la strongyloüdose. Paris, 1935.

Essmont (L.). - Opisthorchis tenuicollis $(=0$. felineus) en Pologne. Cas observés chez l'homme. Annal. paras., XV, 1937, p. 507-517.

ERhardt (A.). - Chemotherapeutische Untersuchungen an der Opisthorchiasis der Katzen. I. Arch. Schiffs-u. Trop. Hyg., XXXVI, 1932, p. 22-31.

- Testierungsmethode dreiwertiger Antimonpraparate an der Opisthorchiasis der Katze. Arch. Schiffs-u. Trop. Hyg., XXXVII, 1933, p. 131-135.

- Systematik und geographische Verbreitung der Gattung Opisthorchis R. Blanchard 1895, sowie Beitraege zur Chemotherapie und Pathologie der Opisthorchiasis. Ztschr. f. Parasitenk., VIII, 1935, p. 188-225.

- Testierungsmethode Arcylostoma-wirksamer Prieparate und chemotherapeutische Untersuchungen an der Ancylostomiasis der Katze. Arch. Schiffs-u. Trop. Hyg., XLII, 1938, p. 108-117.

Graham (G. L.). - Studies on Strongyloides. I. S. ratti in parasitic series, each generation in the rat established with a single homogonic larva. Am. Journ. Hyg., XXIV, 1936, p. 71-87. 
Gratiam (G. L.). - Studies on Strongyloides. II. Homogonic and heterogonic progeny of the single, homogonically derived $S$. ratti parasite. Am. Journ. Hyg., XXVII, 1938, p. 221-234.

- Studies on Strongyloides. III. The fecundity of single S. ratti of homogonic origin. Journ. Paras., XXIV, 1938, p. 233-243.

Langeron (M.). - Précis de microscopie, $5^{e}$ éd., Paris, Masson et Cie, 1934; ef. p. 831-833.

SHELdon (A. J.). - Studies on active acquired resistance, natural and artificial, in the rat to infection with Strongyloides ratti. Journ. Paras., XXII, 1936, p. 533. Am. Journ. Hyg., XXV, 1937, p. 53-65.

Successful infection of mice with Strongyloides ratti. Jourr. Paras., XXIII, 1937, p. 98.

The rate of loss of worms (Strongyloides ratti) in rats. Am. Journ. Hyg., XXVI, 1937, p. 352-354.

Age resistance in laboratory rats to infection with Strongyloides ratti. Am. Journ. Hyg., XXVI, 1937, p. 355-357.

Studies on routes of infection of rats with Strongyloides ratti. Am. Journ. Hyg., XXVI, 1937, p. 358-373.

Infection of an abnormal host (guinea pig) with Strongyloides ratti. Am. Journ. Hyg., XXVII, 1938, p. 298-300.

Zschuске (J.). - Eine Kammer fuer die mikroskopische Zaehlung von Helmintheneiern und-larven. Arch. Schiffs-u. Trophyg., XXXv, 1931, p. 357363.

Institut de Parasitologie de la Faculté de médecine de Paris (Directeur: Prof. E. Brumpt). 